\title{
A New Progressive Coding Algorithm of Dithered Images
}

\author{
Soo-Chang Pei*, Fellow, IEEE, Jing-Ming Guo*, and Hua Lee, Fellow, IEEE \\ *Department of Electrical Engineering \\ National Taiwan University \\ Taipei, Taiwan, R.O.C. \\ E-mail: pei@cc.ee.ntu.edu.tw, \\ ${ }^{+}$Department of Electrical and Computer Engineering \\ University of California \\ Santa Barbara, California 93106-9560, U.S.A \\ E-mail: hualee@ece.ucsb.edu
} imguo@seed.net.tw

\begin{abstract}
A novel progressive coding scheme is presented for the efficient display of dithered images. Dithered images are the results of thresholding original graylevel images with dithering screens. After the preprocessing of bit-interleaving, this algorithm utilizes the characteristic of reordered image to determine the transmitting order and then progressively reconstructs the dithered image. Moreover, the dithered images are further compressed by lossy and lossless procedures. The experimental results demonstrate high-quality reconstructions while maintaining low transmitted bit rates.
\end{abstract}

\section{Introduction}

Digital halftoning is a technique of converting multitone images into the 2-tone format [1]. Conventional halftoning methods include ordered dithering, error diffusion, and least-square methods $[2,3]$. And the ordered-dithering method is known to be the most efficient and offers good visual quality.

Consider the case that a user is to select one from a number of images in a remote database. Assume the images are sequentially compressed prior to transmission. The lossless compression required gives an average compression ratio ranging from 1 to 2 . In narrow bandwidth wireless environment, the compression ratio will not be practical. So, a more effective approach is to use progressive coding to transmit an approximation of the images first at low bit rates. If the user identifies the image of interest, further refinement can be requested.

Kollias and Anastassiou proposed a progressive coding scheme for error-diffused halftone images using a distortion criterion [4]. In this paper, we present a novel progressive coding scheme for dithered images. The performance of the algorithm is measured by PSNR, i.e., the peak signal power to the mean squared error (MSE) between the original dithered image and the reconstructed dithered image at every step. Experimental results show quality halftone reconstruction and high lossy and lossless compression ratios can be achieved.

\section{Bit-interleaving}

There are several kinds of halftone screens, each of which is used for a specific purpose. Fig. 1(a) is a dispersed-dot dithering screen. We now briefly describe the algorithm of ordered dithering. Without loosing generality, we define the size of the halftone screen as $M \times N$. Each pixel $x_{i, j}$ in the original gray level image is mapped to a halfone screen value $H S_{i \bmod M, j \bmod N}$. The dithered output $b_{i, j}$ is determined as

$$
b_{i, j}=\left\{\begin{array}{lll}
255(\text { white }) & \text { if } & x_{i, j} \geq H S_{i \bmod M, j \bmod N} \\
0(\text { black }) & \text { if } & x_{i, j}<H S_{i \bmod M, j \bmod N}
\end{array}\right.
$$

After processing the original gray scale image in Fig. 1(b) with halftone screen in Fig. 1(a), the dithered Lena image $(512 \times 512)$ is shown in Fig. $1(\mathrm{c})$. The bitinterleaving [5] extracts and gathers all the pixels that map to the same threshold in Fig. 1(a) together to form a sub-image, and then resort every sub-images from left to right, bottom to top corresponding to the threshold values from lower to higher level in Fig. 1(a) to form the image shown in Fig. 1(d), which composes 64 sub-images.

\section{Progressive coding with lossy and entropy coding}

\subsection{Progressive coding}

A two-dimensional image such as Fig. 1(d) can be rearranged into the form of a one-dimension data 
sequence, as in Fig. 2(a). Sub-images 1 to 8 are all white while sub-images 57 to 64 are all black. Thus, the pixel values of these sub-images need not be transmitted, because the receiver recognizes it when it receives an overhead bit stream of 64 bits. In this bit stream, the bit " 1 " appears in the interval $(1,32)$, which represents an all-white sub-image. On the other hand, the bit " 1 " appears in the interval $(33,64)$, representing an all-black sub-image. By doing so, we can reduce a quarter of data set in this example and the receiver can reconstruct it by filling all white (or black) in the relative positions of image after receiving this bit stream.

Fig. 1(d) shows that the sub-images in position "32" or " 33 " preserve a significant portion of the original image's features. In other words, the sub-images in position "32" or " 33 " should transmitted and reconstructed first (in this paper we use 32). The receiver reconstructs the image by duplicating it and filling in positions 9 to 56 , and then conduct inverse bit-interleaving.

The algorithm for the determination of the subsequent sub-images to be transmitted is described as follows.

1. Let $x_{1}, x_{2}, \ldots, x_{m}$ represent the one-dimension sub-images. $\left(\mathrm{m}=n^{2}\right.$, here $\left.\mathrm{n}=8, \mathrm{~m}=64\right)$

2. Initialize flag $\left(x_{1}\right)=\operatorname{flag}\left(x_{2}\right)=\ldots=\operatorname{flag}\left(x_{m}\right)=0$.

3. Set flag $\left(x_{\text {upper }}\right)=\operatorname{flag}\left(x_{\text {lower }}\right)=1 .\left(x_{\text {lower }}\right.$ represents the sub-image before the sub-image that a black pixel first appears if counting from $x_{1}$, where $x_{\text {upper }}$ represents the sub-image before the subimage that the white pixel first appears if counting down from $x_{m}$. In the example, the $256 \times 256$ Lena image, $x_{\text {lowier }}=x_{8}, x_{\text {uppet }}=x_{57}$ )

4. Set flag $\left(x_{\text {middle }}\right)=1$, here $x_{\text {middle }}$ represents $x_{32}$ in this paper $\circ$

5. Calculate the difference $D_{a b}$ of every two adjacent sub-images $x_{a}^{*}, x_{b}$ such that $\operatorname{flag}\left(x_{a}\right)=\operatorname{flag}\left(x_{b}\right)=$ 1 , define $D_{a b}=D_{a b}{ }^{(1)}+D_{a b}{ }^{(2)}$, where $D_{a b}{ }^{(1)}=\mathrm{H}\left(x_{a}, x_{a+1}\right)+\mathrm{H}\left(x_{a}, x_{a+2}\right)+\ldots+\mathrm{H}\left(x_{a}, x_{c}\right)$, $D_{a b}{ }^{(2)}=\mathrm{H}\left(x_{c+1}, x_{b}\right)+\mathrm{H}\left(x_{c+2}, x_{b}\right)+\ldots+$ $\mathrm{H}\left(x_{b-1}, x_{b}\right), \mathrm{a} \leq \mathrm{c} \leq \mathrm{b}$

$D_{a b}{ }^{(1)}, D_{a b}{ }^{(2)}$ satisfy the condition $D_{a b}{ }^{(1)} \cong D_{a b}{ }^{(2)} \cdot\left(\mathrm{H}\left(\boldsymbol{x}_{a}, x_{a+1}\right)\right.$ represents the hamming distance between $x_{a}$ and $x_{a+1}$ ). Then $x_{c}$ is the next sub-image to be transmitted, and set $\operatorname{flag}\left(x_{c}\right)=1$.

6.The reconstructed left-side distance (LSD) and rightside distance (RSD), which satisfy the following conditions:

$H\left(x_{a}, x_{a+1}\right)+H\left(x_{a}, x_{a+2}\right)+\ldots+$ $H\left(x_{a}, x_{c-L S D-1}\right) \cong H\left(x_{c-L S D}, x_{c}\right)+H\left(x_{c-L S D+1}, x_{c}\right)$

$+\ldots+H\left(x_{c-1}, x_{c}\right)$

$H\left(x_{c}, x_{c+1}\right)+H\left(x_{c}, x_{c+2}\right)+\ldots+$ $H\left(x_{c}, x_{c+R S D}\right) \cong H\left(x_{c+R S D+1}, x_{b}\right)+H\left(x_{c+R S D+2}, x_{b}\right)+$ $\ldots+H\left(x_{b-1}, x_{b}\right)$

LSD and RSD are then represented by five bits and transmitted to the receiver as side information.

\subsection{Lossy processing and entropy coding}

In the previous section, we illustrated the procedure of progressive transmission for lossless reconstruction of the original dithered images. In some practical applications, perfect reconstruction may not be as important as the processing speed. Thus, it is of importance to analyze the trade-off between reconstruction errors and processing speed. Here in this section, we provide the overview of the algorithm for the improvement of processing speed with a lossy process, as follows:

1. Define a threshold $N_{t h}$, as the total number of pixels of which the values can be changed in a reconstructed progressive dithered image.

2. Find the minimum minor pixel (minor pixel means black pixel in $x_{1} \sim x_{\text {middle }}$ or white pixel in $x_{\text {middle }+1} \sim x_{m}$ ) number of the sub-image simultaneously searching up from $x_{1}$ and down form $x_{64}$.

3. Reverse a minor pixel value (white to black, or black to white) of the sub-image with minimum minor pixel as given in Step 2 and then subtract 1 from $N_{t h}$.

4. Repeat Steps 2 and 3 until $N_{t h}=0$ (when $N_{t h}=0$ means already have amount of $N_{t h}$ pixels changed in a dithered image).

Thus we can produce a bit-interleaved image with more all black (white) sub-images, or generally speaking, an image with lower entropy. 
Finally, we employ a lossless Huffman coder to perform further entropy coding of sub-images to be transmitted. Because the pixels are average white in the lower half plane and black in the upper half plane, as shown in Fig. 1(d), the coding gain can be improved if we separated the Huffman coding process into the lower plane and upper plane.

\section{Experimental results}

A standard $256 \times 256$ pixel 8 -bits gray-tone image of Lena, as shown in Fig. 3(a), is used as the test image of the experiments. The results of progressive reconstruction are shown in Figs. 3 (b)-(h), corresponding to step 1 to step 7, which are the reconstructions from $1,2,4,8,16,32$, and 48 subimages. Figure $3(\mathrm{~h})$ is the final reconstruction from all 48 sub-images. This is because there are 16 all black and all white sub-images and only a bit stream will be required to transmit to represent the images.

The images of Peppers, Mandrill, Milk, and Airplane were also used for the experiments for comparison purposes, and the experimental PSNR corresponding to the number of steps is shown in Fig. 4. The quality of the reconstructions showed notable improvement over the resultant images by the technique proposed by Kollias [4].

The average Huffman lossless compression bit rate is 0.34 , while the resultant bit rate is 0.75 by Kollias [4]. The bit rates of the progressive lossy coding of the Lena image described in Section III(B) under 400, 800, 1200 pixels' loss are $0.3,0.29$, and 0.26 , as well as the PSNR are 22.14, 19.13, and 17.37, respectively. Figs. 5 (a)-(c) are corresponding to $400,800,1200$ pixels' loss, respectively, at $150 \mathrm{dpi}$.

\section{Conclusions}

In this paper, a new progressive coding algorithm for dithered images was presented. The experimental results showed high-quality reconstruction of dithering images. In addition, the lossy and entropy coding procedure preserved the image quality at low coding rates. In the paper, the algorithm was applied to dispersed-dot dithering images. Nonetheless, it can be modified and generalized to include clustered-dot dithering images. The performance of the algorithm can be further improved by incorporating the behavior of the human visual system.

\section{References}

[1] R. Ulichney, Digital Halftoning, MIT Press, Cambridge, Massachussetts, 1987.
[2] R. W. Floyd and L. Steinberg, "An adaptive algorithm for spatial gray scale," Digest. Society for Information Display, pp.36-37, 1975.

[3] M. Analoui, and J. P. Allebach, "Model based halftoning using direct binary search," Proc. SPIE, Human Vision, Visual Proc., Digital Display III, vol. 1666, pp. 96-108, Feb. 1992.

[4] S. Kollias and D. Anastassiou,"A progressive scheme for digital image halftoning, coding of halftones, and reconstruction," IEEE Transactions on Selected Areas in Communications, vol. 10, pp. 944-951, 1992.

[5] C. N. Judice, "Data reduction of dither coded images by bit interleaving," Proc. Soc. Inform. Display, vol. IT-17(2), pp. 91-97, 1976. 


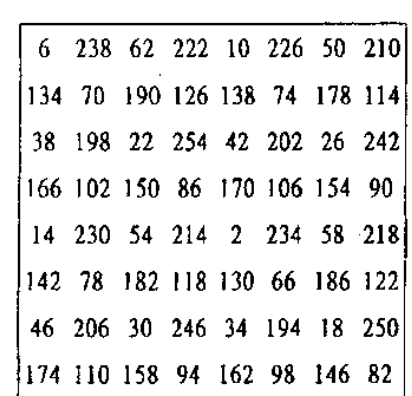

(a)

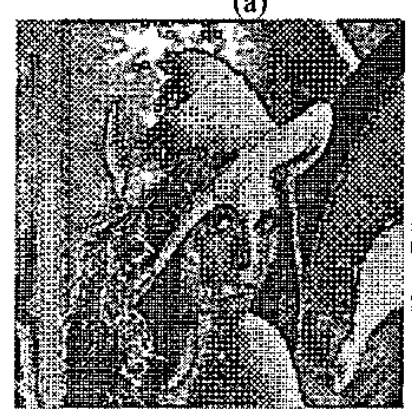

(c)

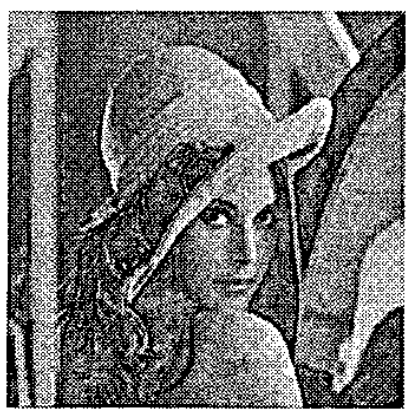

(b)

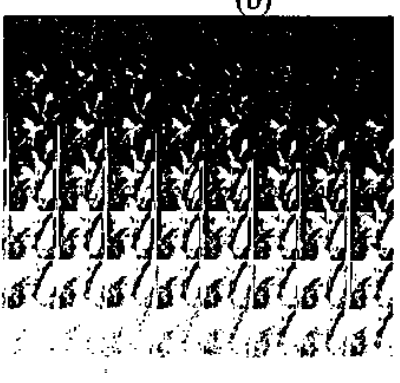

(d)

Fig. 1. (a) $8 \times 8$ halftone screen (b)Original $256 \times 256$ gray scaled Lena image. (c)Dithered image. (d) After bit-interleaving. (all printed at $150 \mathrm{dpi}$ )

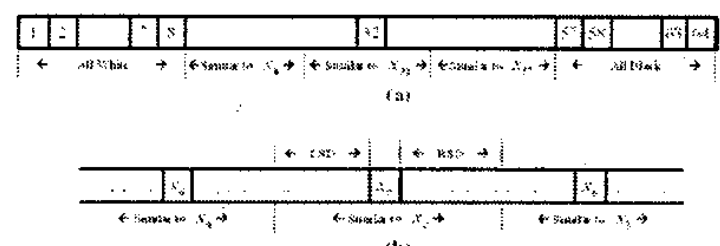

Fig. 2. 1-D representation of subimages. (a)Order of 64 sub-images. (b)LSD and RSD of $x_{c}$

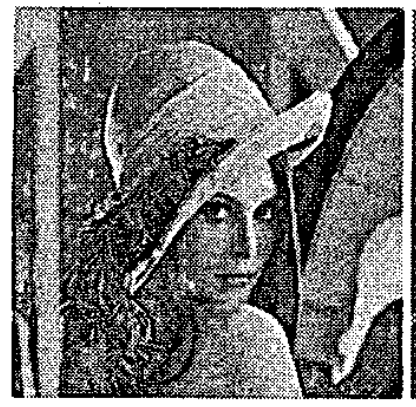

(a)

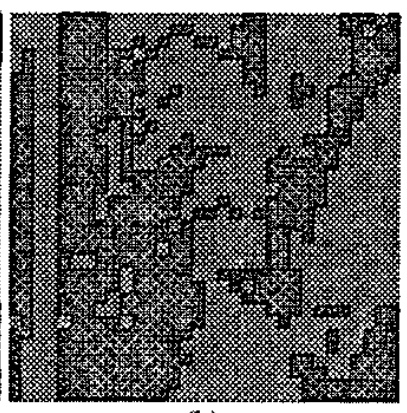

(b)

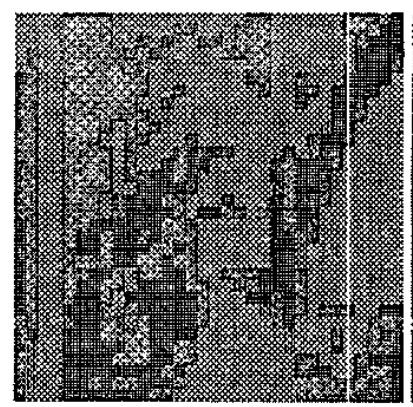

(c)

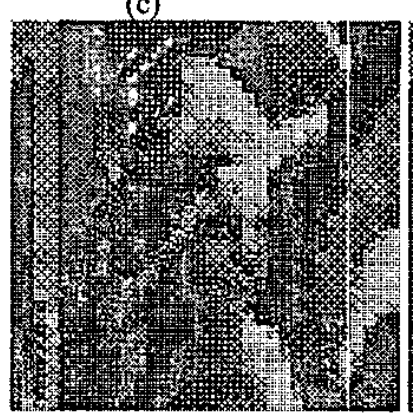

(e)

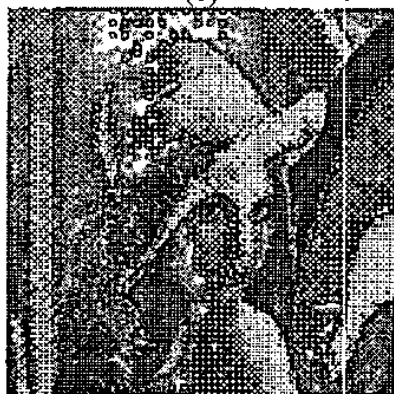

(g)

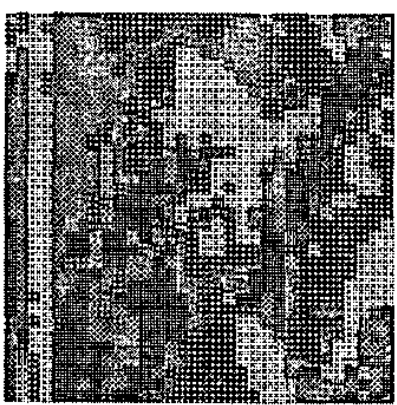

(d)

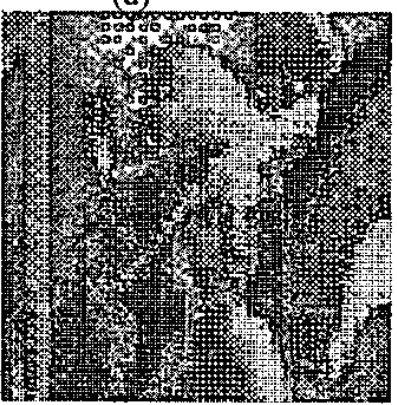

(f)

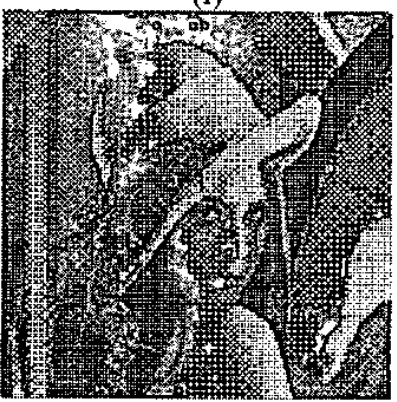

(h)

Fig. 3 (a)Original $256 \times 256$ gray-scaled Lena image. (b)-(h) Reconstructed dithering $256 \times 256$ Lena images in 7 steps (all printed at $150 \mathrm{dpi}$ ).

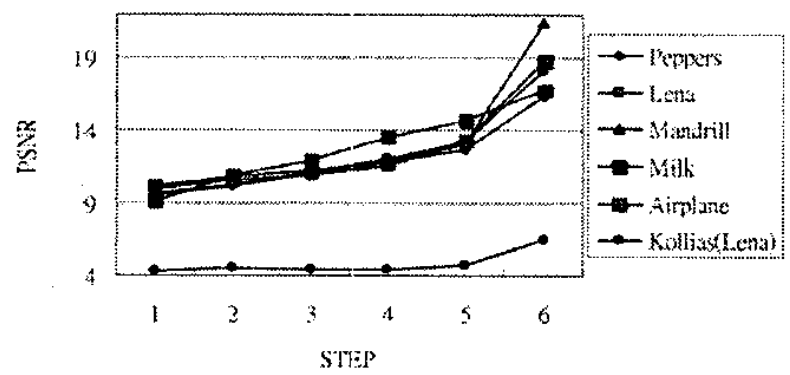

Fig. 4. PSNR v.s. Reconstructed steps of five tested images 


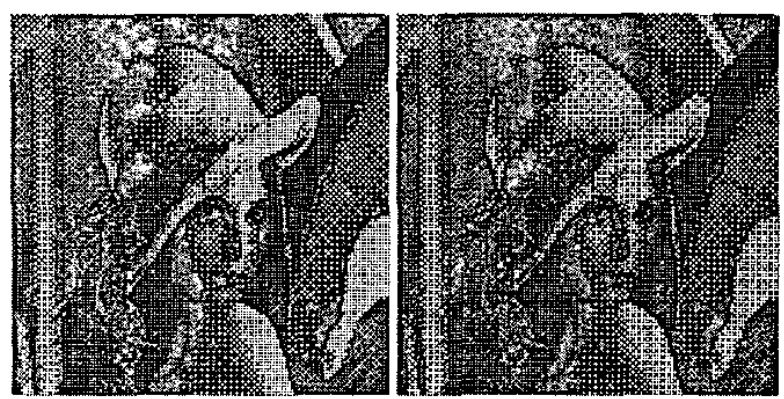

(a)

(b)

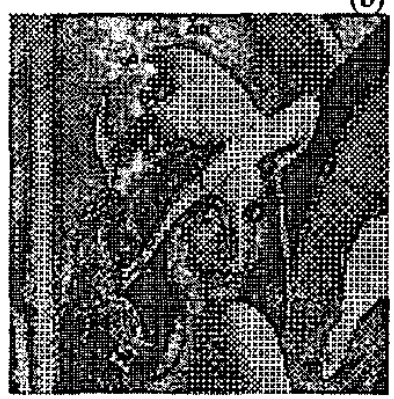

(c)

Fig. 5. Lossy processing results $400,800,1200$ pixels' loss. 